\title{
Numerical Simulation of Temperature Field in Selective Laser Sintering
}

\author{
Jian Zhang, Deying Li ${ }^{*}$, Jianyun Li, and Longzhi Zhao \\ Key Laboratory of Ministry of Education for Conveyance and Equipment, \\ East China Jiaotong University, Nanchang 330013, China \\ zhj6115@126.com, lideyingecjtu@163.com, \\ shixinyu841031@126.com, zhaolongzhi@163.com
}

\begin{abstract}
The laser sintering process of multi-component powder $\mathrm{W} / \mathrm{Cu}$ is simulated by ANSYS software based on the factors of radiation, convection and thermal physical parameters on temperature. The laser power and scanning velocity which are the key process parameters to affect directly in sintering molding are studied in paper. The results show that when the scanning velocity is constant, the sintering depth is rising with the increase of laser power; In addition, when the laser power is constant, the sintering depth is decreasing with the increase of scanning velocity. To select reasonable processing parameters and meet the requirements of sintering quality on the sintering depth, the parameters of laser power and scanning velocity are optimized by analyzing the sintering depth.
\end{abstract}

Keywords: Selective laser sintering, Numerical simulation, Temperature field, Sintering depth.

\section{Introduction}

W/Cu composite, a typical two-phase pseudo alloy, exhibits excellent electrical conductivity and thermal conductivity of $\mathrm{Cu}$ and high melting point and high hardness of W. It can be used as electrical contact and electrode material, military material for special purpose (such as rocket nozzle), and heat sink substrate with high air tightness packing of CPU and solid-state microwave tube, etc. So W/Cu composite has been widely used both in industry and military $[1,2]$.

Selective Laser Sintering (SLS), which becomes a research focus recently, can manufacture directly the metal mold or parts prototype with the complex shape from 3D CAD model, to obtain the functional parts meeting the requirements. For the advantages of short manufacturing cycle, wide range of materials and others, SLS is expected to obtain the $\mathrm{W}-\mathrm{Cu}$ components with high density and complex shape that other process is difficult to do. In this paper, the temperature field distribution of W-Cu mixed powder is studied by ANSYS numerical simulation, and the effects of laser

${ }^{*}$ Corresponding author. Tel.: 13707096493.

D. Li, Y. Liu, and Y. Chen (Eds.): CCTA 2010, Part IV, IFIP AICT 347, pp. 474 479, 2011.

(c) IFIP International Federation for Information Processing 2011 
power and scanning velocity on sintering depth are analyzed. The reasonable processing parameters are selected by sintering depth, which has great significance for the sintering process of $\mathrm{W}-\mathrm{Cu}$ mixed powder.

\section{Establishment of Model}

\subsection{Establishment of Mathematic Model}

The molten pool has heat change with the surrounding air and powder bed in SLS, and the thermal conduction can be expressed by equation (1) [3].

$$
k_{e}\left(\frac{\partial T^{2}}{\partial x^{2}}+\frac{\partial T^{2}}{\partial y^{2}}+\frac{\partial T^{2}}{\partial z^{2}}\right)+q_{c}+q_{g}=\rho C \frac{\partial T}{\partial t}
$$

Where $k_{e}$ is effective thermal conductivity of powder; $\rho$ is compacted density of powder; $\mathrm{C}$ is heat capacity of material; $q_{c}$ is dispersed heat to air; $q_{g}$ is laser power density.

Assume that the initial temperature T0 of powder bed is evenly distributed before sintering, and the initial condition is shown as follows:

$$
\left.T(x, y, z, t)\right|_{t=0}=T_{0}
$$

In order to simplify calculation, assume that there is no heat loss on the bottom of substrate. In SLS, there is heat dissipation by convection and radiation, the boundary condition is shown as follows.

$$
-\left.k_{e} \frac{\partial T}{\partial z}\right|_{z=0}+h\left(T-T_{E}\right)+\sigma \varepsilon\left(T^{4}-T_{E}^{4}\right)=q
$$

Where $k_{e}$ is effective thermal conductivity of powder; $h$ is heat convection coefficient; $T$ is temperature of metal powder at a certain moment; $\sigma$ is Stefan-Boltzmann constant; $T_{E}$ is initial temperature; $\varepsilon$ is heat radiation coefficient of actual objects.

\subsection{Establishment of Physical Model}

In order to simplify the sintering model and reduce the computing time, the finite element model is established in Fig. 1. The multi-component powder consist of $\mathrm{W}$ and $\mathrm{Cu}$ with a certain percentage, the size of which is $3.4 \mathrm{~mm} \times 1.6 \mathrm{~mm} \times 0.3 \mathrm{~mm}$. To ensure 
the enough mesh density in laser heating zone and heat affected region and reduce time, the different meshing sizes can be adopted to the substrate and sintered layers. The meshing of sintered layers is smaller $(0.1 \mathrm{~mm})$, while the meshing of substrate is larger.

The Gaussian energy distribution of laser is shown in Figure 2, the laser energy of center is the highest, and the laser energy is continuously reduced away from the spot center. The laser spot is divided into $4 \times 4$, as shown and the decay coefficients of $1,2,3$ are $0.8359552,0.5226424,0.3267576[4]$.

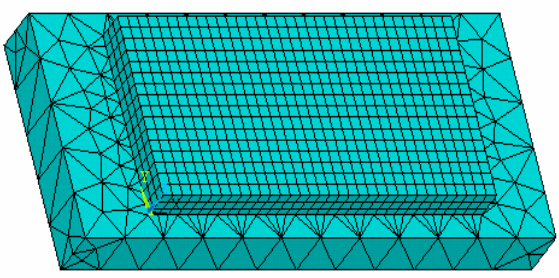

Fig. 1. Finite element model

\begin{tabular}{|l|l|l|l|}
\hline 3 & 2 & 2 & 3 \\
\hline 2 & 1 & 1 & 2 \\
\hline 2 & 1 & 1 & 2 \\
\hline 3 & 2 & 2 & 3 \\
\hline
\end{tabular}

Fig. 2. Gaussian energy distribution of laser

In the sintering process, the laser energy obeys the Gaussian distribution.

$$
q=\frac{2 A P}{\pi \varpi^{2}} \exp \left(-2 \frac{r^{2}}{\varpi^{2}}\right)
$$

Where $q$ is laser power density; $p$ is laser power; $A$ is absorption rate of powder bed to laser; $r$ is distance between any point on the powder bed and laser spot center; $\omega$ is radius of laser spot.

\section{Results and Analysis}

The powder consists of $\mathrm{W}$ and $\mathrm{Cu}$ with a certain percentage, the melting points are $3380^{\circ} \mathrm{C}$ and $1083^{\circ} \mathrm{C}$, and the other thermal physical properties on temperature are shown in Table 1 and Fig. 3.

The process parameters to simulate that the spot diameter is $0.4 \mathrm{~mm}$, the scanning spacing is $0.3 \mathrm{~mm}$, the powder thickness is $0.1 \mathrm{~mm}$, the convection coefficient of air is 10 , the effective radiation coefficient of powder bed is 0.8 .

Table 1. Thermal physical properties of $\mathrm{Cu}$

\begin{tabular}{ccccccccc}
\hline $\mathrm{T} /{ }^{\circ} \mathrm{C}$ & 20 & 100 & 200 & 400 & 500 & 600 & 800 & 1000 \\
\hline $\mathrm{C} / \mathrm{J}(\mathrm{kg} \cdot \mathrm{k})^{-1}$ & 385 & 388 & 392 & 400 & 409 & 414 & 420 & 445 \\
$\mathrm{~K} / \mathrm{W}(\mathrm{m} \cdot \mathrm{k})^{-1}$ & 405 & 404 & 399 & 379 & 355 & 342 & 329 & 313 \\
\hline
\end{tabular}




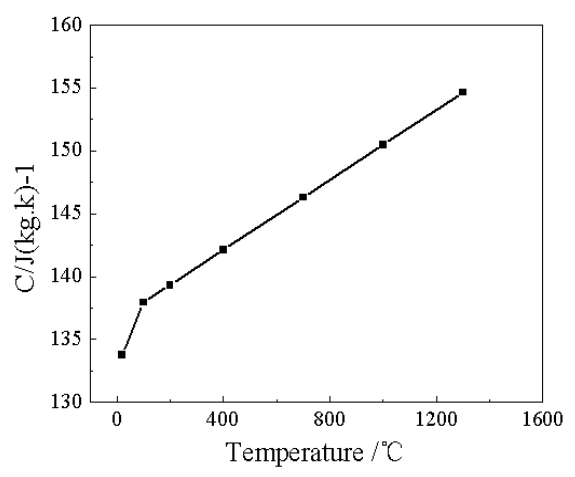

(a) Specific heat capacity

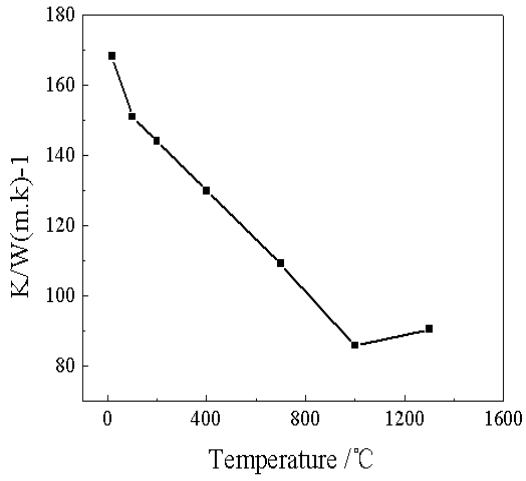

(b)Thermal coefficient

Fig. 3. Thermal physical properties of W

Fig. 4 shows the effects of scanning velocity on pool depth. As seen from it, when the laser power is constant, the sintering depth is decreasing with the increase of scanning velocity. The sintering depth is $0.29 \mathrm{~mm}$ at $\mathrm{v}=0.12 \mathrm{~m} / \mathrm{s}$, while only is the sintering depth $0.076 \mathrm{~mm}$ at $\mathrm{v}=0.20 \mathrm{~m} / \mathrm{s}$. The reason is that when the laser power is constant and the scanning velocity is increasing, the input laser energy decreases. Meanwhile, the time that energy on the powder particles is decreasing. Especially at high scanning speed, the contact time between energy and powder particle is very short, even the powder particles could not be melt for the energy transfer around the powder particles without enough time. But the temperature is rising quickly at low scanning speed, which leads easily to greater heat accumulation effect, and results in larger temperature gradient.

The effects of laser power on pool depth are shown in Fig. 5. As seen from it, when the scanning velocity is constant, the sintering depth is rising with the increase of laser power. The sintering depth is only $0.08 \mathrm{~mm}$ at $\mathrm{p}=195 \mathrm{w}$, and the sintering depth is
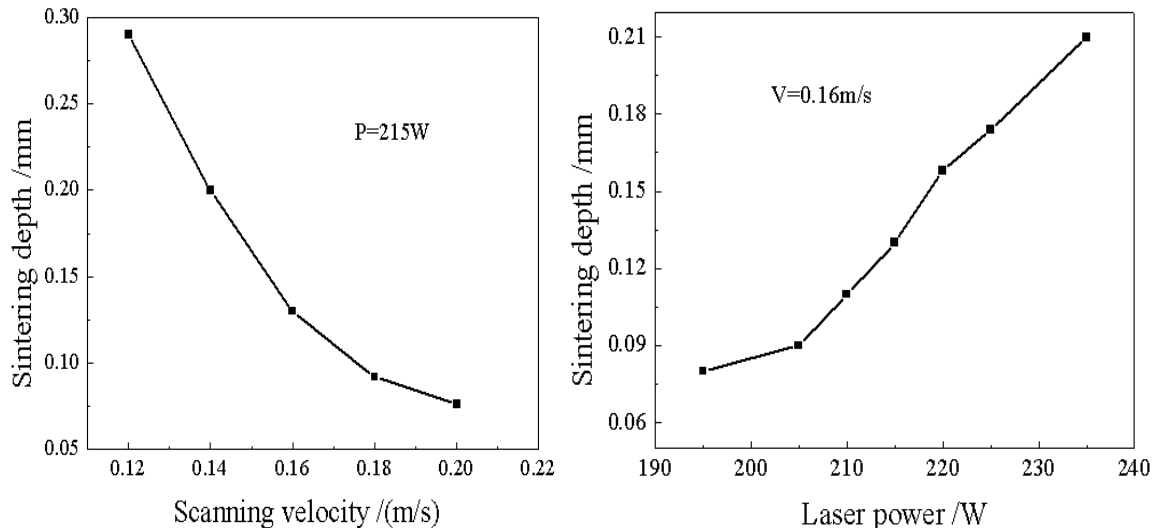

Fig. 4. Effects of scanning velocity on pool depth Fig. 5. Effects of laser power on pool depth 
increasing to $0.21 \mathrm{~mm}$ at $\mathrm{p}=235 \mathrm{w}$. The reason is that when the scanning velocity is constant and the laser power is increasing, the output energy density is rising. But the excessive laser power makes the temperature so high that the powder layers have a greater contraction, and is easier to warping and cracking.

The laser power and scanning velocity are the key process parameters to affect directly in sintering molding. The higher scanning velocity can not only effectively suppress the expansion of molten pool and the phenomenon of "splashing", but also improve the forming efficiency. The reasonable laser power can not only ensure the layers bond well, but also reduce the contraction and warping.

The sintering depth must be greater than the powder thickness, to ensue the adjacent layers bond well by penetrating the current layer. Otherwise the adjacent layers will be separated, and the poor strength and accuracy of forming will be obtained, even the forming could not be completed. Fig.6 shows the temperature distribution of Z-direction at different time with a certain laser power and scanning velocity. As seen from it, the temperature of $0.1 \mathrm{~mm}$ is obviously higher than the melting point of $\mathrm{Cu}$ $(1356 \mathrm{~K})$ at $\mathrm{t}=0.05875 \mathrm{~s}$, it indicates that the sintering depth is greater than the powder thickness. Similarly, the sintering depth are about $0.14 \mathrm{~mm}$ and $0.19 \mathrm{~mm}$ at $\mathrm{t}=0.11125 \mathrm{~s}$ and $t=0.21125 \mathrm{~s}$, so the powder thickness is less than the sintering depth. At the current process parameters, the related joint between the first layer and substrate is strong, and the metallurgical bonding of layers is well.

The 3D parts in SLS are completed by lines to surface and layer by layer overlapped, and the following two conditions should be met to form solid structure by sintering $[5,6]$.

1) The degree coverage of energy should be required between scanning beams, that is, the scanning velocity could not be too high with a certain power density, ensure that the enough power input to make the powder in the current layer bond together and form the 2D surface structure without loose powder.

2) The powder thickness is less than the sintering depth, that is, the laser power should ensure the adjacent layers bond well by penetrating the current layer to form the $3 \mathrm{D}$ body structure.



Fig. 6. Temperature distribution of Z-direction at different time $(1-\mathrm{t} 1=0.05875 \mathrm{~s}, 2-\mathrm{t} 2=0.11125 \mathrm{~s}$, $3-\mathrm{t} 3=0.21125 \mathrm{~s})$ 


\section{Conclusion}

1) When the scanning velocity is constant, the sintering depth is rising with the increase of laser power; In addition, when the laser power is constant, the sintering depth is decreasing with the increase of scanning velocity.

2) To select reasonable processing parameters and meet the requirements of sintering quality on the sintering depth, the parameters of laser power and scanning velocity are optimized by analyzing the sintering depth.

Acknowledgement. This work was sponsored by Science and Research Foundation of East China Jiaotong University (01308013), the Natural Science Foundation of Jiangxi Province (09497, 2009GQC0014, 2008GZC0037) and Graduate Innovation Foundation of East China Jiaotong University (YC09C002).

\section{References}

1. Liu, T., Fan, J.L., Tian, J.M.: Synthesis and Sintering of Ultra-fine/nanometer W-10\%Cu Composite Powder. Journal of Central South University (Science and Technology) 40(5), 1235-1239 (2009)

2. Gu, D.D., Shen, Y.F.: Microstructures of Laser Sintered Micron/nano-sized Cu-W Powder. Acta Metallurgica Sinica 45(1), 113-118 (2009)

3. Zhang, W.X., Shi, Y.S., Li, J.G.: Simulation of Temperature Field for Optimization of Processing Parameters of Selective Laser Melting Metal Powders. Applied Laser 28(3), 185-189 (2008)

4. Ma, L., Huang, W.D., Yu, J.: Parametric Finite Element Model of Temperature/stress Field Evolution by Metal Laser Solid Forming. Chinese Journal of Laser 36(12), 3227-3232 (2009)

5. Kumar, S., Chatterjee, A.N., Saha, P.: An experimental design approach to selective laser sintering of low carbon steel. Journal of Materials Processing Technique 136(1/3), 151-157 (2003)

6. Tolochko, N.K., Mozzharov, S.E., Yadroitsev, I.A.: Selective laser sintering and cladding of single-component metal powders. Rapid Prototyping Journal 10(2), 88-97 (2004) 